LA-UR- $96-992.90 N-95 / 0 / 06--4$
Title: $\mid \begin{aligned} & \text { MEASUREMENTS OF NONLINEAR INDEX BY A RELAY- } \\ & \text { IMAGED TOP-HAT Z-SCAN TECHNIQUE }\end{aligned}$

Author(s):

T. Shimada, P-24

N. A. Kurnit, P-24

M. Sheik-Bahae, UNM
RECEIVED APR 011996

OSTI

Submitted to:

Annual Symposium on Optical Materials for High Power Lasers October 30 - November 1, 1995

Boulder, $\mathrm{CO}$

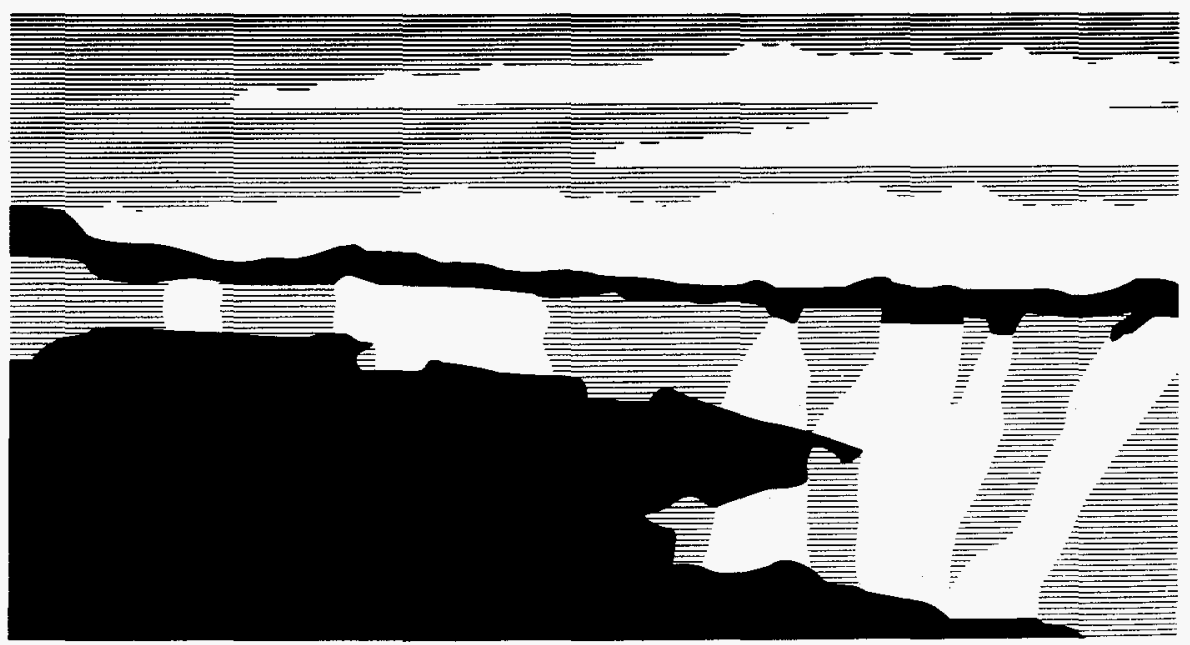

Los Alamos National Laboratory, an aftirmative action/equal opportunity employer, is operated by the University of California for the U.S. Department of Energy under contract W-7405-ENG-36. By acceptance of this article, the publisher recognizes that the U.S. Government retains a nonexclusive, royalty-free license to publish or reproduce the published form of this contribution, or to allow others to do so, for U.S. Government purposes. The Los Alamos National Laboratory requests that the publisher identify this article as work performed under the auspices of the U.S. Department of Energy. 


\title{
Measurement of nonlinear index by a relay-imaged top-hat Z-scan technique
}

\author{
T. Shimada and N. A. Kurnit \\ Physics Division, Los Alamos National Laboratory \\ MS E526, Los Alamos, New Mexico 87545 \\ and \\ M. Sheik-Bahae \\ Department of Physics and Astronomy, University of New Mexico \\ Albuquerque, NM 87131
}

\begin{abstract}
Measurements of the nonlinear index of a number of materials of interest for the National Ignition Facility have been performed at $1064 \mathrm{~nm}$ and $355 \mathrm{~nm}$ by a modified version of the "top-hat" Z-scan technique and the results compared with the more standard gaussian-beam Z-scan technique. The top-hat technique has the advantages of higher sensitivity and smaller uncertainties introduced by beam-quality considerations. We have made what we feel to be an additional improvement by placing the defining aperture for the top hat at the front focal plane of the lens that focuses the beam into the sample and then reimaging the input aperture with a second lens onto a ccd camera. Reimaging eliminates diffraction fringes and provides a stationary image even for a wedged sample; recording the entire image permits minimization of spurious effects such as varying interference fringes.
\end{abstract}

\section{Introduction}

Accurate values of the nonlinear index, $n_{2}$, of optical materials to be utilized in the National Ignition Facility are needed for specifying the optical components in this facility because of the deleterious effect of the "B" integral, or intensity-dependent phase shift $\Delta \Phi$,

$$
B=\Delta \Phi=\frac{2 \pi}{\lambda} \int \gamma I d z
$$

on beam uniformity, harmonic conversion efficiency, and focusability. This phase shift arises from the change in refractive index with intensity, $n=n_{o}+n_{2}\left\langle E^{2}\right\rangle=n_{o}+\left(n_{2} / 2\right) E_{o}^{2}=n_{o}+\gamma I$, where $n_{2}$ is usually expressed in esu and $\gamma$ in $\mathrm{cm}^{2} / \mathrm{W}$ and are related by $\gamma\left(\mathrm{cm}^{2} / \mathrm{W}\right)=\left(4 \pi \times 10^{7} / \mathrm{cno}_{o}\right) \times n_{2}(\mathrm{esu})$. Although $n_{2}$ for materials of interest is believed to be known with reasonable accuracy at the $1 \mu \mathrm{m}$ fundamental $(1 \omega)$, there exists substantial variation in the measurements that have been made at the second $(2 \omega)$ and third $(3 \omega)$ harmonic frequencies, even for so basic a material as fused silica. ${ }^{1,2}$ Measurements made by different techniques varied by as much as a factor of two. In surveying the techniques that had been utilized for $n_{2}$ measurements, we concluded that beam distortion measurements, 
as made by the $\mathrm{Z}$-scan method, ${ }^{3}$ offered a relatively direct measure of the peak phase shift experienced by a beam propagated through a nonlinear medium, and that the technique had been refined to a level that appeared to yield good accuracy.

In the standard Z-scan method, a sample is scanned through the focus of a gaussian beam and the energy passed by an aperture placed in the far field is measured as a function of sample position ( $z$ ). For a sample placed in front of the focus, the sample acts (in the case of a positive nonlinear index) to focus the beam more tightly, leading to greater divergence in the far field, and a decrease in the central intensity passed by the aperture. The decrease in intensity becomes greatest (for a sample thin compared to the Rayleigh range of the beam) when the sample is centered at $0.86 z_{o}$, where $z_{o}=\pi w_{o}^{2 / \lambda}$ is the free-space Rayleigh range. ${ }^{3}$ As the sample is moved through the focus, the intensity passed by the aperture returns to the value for the sample far from the focus and then increases to a maximum when the sample is at $0.86 z_{o}$ beyond the focus, at which point the beam experiences the maximum amount of convergence induced by the nonlinear lensing. The intensity passed by the aperture thus displays a characteristic dispersion shape, which for a small aperture centered on a gaussian beam can be shown ${ }^{4,3}$ to have a peak-to-valley transmission difference, $\Delta T_{p v}=\left(I_{p}-I_{v}\right) / I_{o}$ given by

$$
\Delta T_{p v}=0.406 \Delta \Phi_{G}
$$

where $I_{p}, I_{v}, I_{o}$ are, respectively, the on-axis far field intensities measured with the sample at the peak, valley, or far from focus, and $\Delta \Phi_{G}$ is the peak phase shift experienced by the gaussian beam for a sample thin compared to $z_{o}$ placed at the focus.

A variation on this technique has recently been demonstrated 5 in which the input beam is expanded and apertured so as to have a circular flat top distribution in front of the focusing lens and an Airy distribution at the focus. ${ }^{6}$ The rather surprising result of Ref. 5 is that, if the intensity of this top-hat (TH) input beam is adjusted to give the same focused intensity as the gaussian beam, and hence the same peak phase shift (for a thin sample), $\Delta \Phi_{T H}=\Delta \Phi_{G}$, then the change in transmission at the peak and valley is 2.5 times greater than for the gaussian beam. The size of the focal spot can be made comparable to (actually about $14 \%$ smaller in diameter than) that of the original gaussian beam by choosing the aperture diameter to be $d=\pi w_{1}, 5$ where $w_{1}$ is the $1 / \mathrm{e}^{2}$ radius of the gaussian beam at the focusing lens. This gives an intensity distribution at the focus of a lens of focal length $f$ that can be written in terms of the first order Bessel function $J_{1}$ as $I_{o}\left[2 J_{1}\left(\pi r / w_{o}\right) /\left(\pi r / w_{o}\right)\right]^{2}$, with $w_{o}=\lambda f / d$ having the same value as the waist of the focused gaussian and $I_{o}=I_{1}\left(\pi d^{2} / 4\right)^{2} /(\lambda f)^{2}$, where $I_{1}$ is the intensity at the input aperture. The Z-scan curve then has its maximum and minimum at very nearly the same values of $\mathrm{z}$ as the gaussian curve, although the width of the curve is narrower as a result of the rapidly changing intensity and phase distribution in the region of the focus. ${ }^{6}$ Thus not only is the signal size larger for the same focal spot intensity, but the dispersion-like curve is sharper.

The other major advantage of this top-hat technique is that it is possible to make a good approximation to a flat-top intensity (and phase) distribution as long as one has enough energy to expand the beam to a large size, whereas it is not always possible to ensure a perfect gaussian distribution. Our nearly-gaussian beam profile, used for gaussian-beam measurements at $1064 \mathrm{~nm}$, was obtained by 
passing a small portion of a $\mathrm{Nd}$ :YAG output beam through a small aperture and propagating it over a large distance in which it is further apertured, sliced in time by a Pockels cell, and reamplified. Although a good fit to a gaussian ( $-93-97 \%)$ is obtained, it is seen by measuring the spot size by scanning a ccd camera through the focus (Fig.1) that the spot size deviates from the ideal gaussian over a region comparable to $z_{o}$, and attains a minimum value that is typically on the order of $20 \%$ larger than that of the ideal gaussian. Part of this deviation arises from a small amount of astigmatism, part from aperturing the beam at a diameter of approximately $3 w_{1}$ (without which significant departures from gaussian behavior are observed in the focal region, indicating the presence of substantial low-level energy in the wings of the input beam), and part from additional aberrations. This deviation calls into question the validity of the relationship expressed by Eq. 2 . Calculations discussed below show that the results obtained using Eq. 2 are in fact not strictly valid in the presence of beam distortion, but that the results are relatively insensitive to the beam distortion.

\section{Relay-Imaged Top-Hat Z-Scan Technique}

We introduce here a modification to the top-hat $\mathrm{z}$-scan technique in which the defining aperture for the top hat is placed at the front focal plane of the lens of focal length $f_{1}$ which focuses the beam into the sample (Fig. 2). A second lens of focal length $f_{2}$ is placed at a distance $f_{1}+f_{2}$ from the first lens so as to form an image of the aperture at a distance $f_{2}$ from the second lens; this focal length is chosen so as to fill a large fraction of the ccd detector used for measurement of relative intensities in the image. An advantage of this procedure is illustrated in Fig. 3, which compares the calculated intensity distribution without reimaging (Fig. 3(a)) to that obtained with reimaging (Fig. 3(b)). The intensity distibution in the relay-imaged case is seen to go from a flat top to a smoothly varying distribution with depressed center and enhanced edges when the sample is in front of the focus, to one with enhanced center and depressed wings when the sample is after the focus. In the non-imaged case, strong Fresnel diffraction fringes are observed, whose overall pattern in the presence of self focusing (not shown) go up and down in proportion to that observed in the relay-imaged case. This can be a source of additional uncertainty when measuring the central intensity of the beam, whereas in the relay-imaged case the intensity distribution is smooth, but cuts off sharply at the edge of the image of the aperture, allowing precise determination of the beam center and its intensity. Furthermore, if there is a slight wedge in the sample, in the non-imaged case the beam position will translate across the detector as the sample is scanned, but remains stationary in the relay-imaged case. If one were to use an aperture with an energy detector behind it for the Z-scan measurement (as has most often been done), the motion of the beam across the aperture would give a spurious result, particularly in the presence of Fresnel fringes. Our measurements have been made using a ccd camera to record the entire image of the beam; hence small shifts in the beam position are not a problem provided the ccd response is sufficiently uniform. However, we have measured one sample that had a wedge so large that it would have shifted part of the beam off the detector in the absence of image relaying. It should be noted that the beam does move across the second lens in the case of a wedged sample, and reflections from the lens surfaces can lead to interference fringes moving across the image if 
the lens is not antireflection coated.

The experimental setup also employed a low intensity reference arm similar to the sample test arm in which both an image of the aperture and a fraction of the energy, measured or a pyroelectric detector, were recorded. The energy monitor was calibrated against the throughput energy : in the sample arm in the absence of a sample, and was used to select pulses that fell within a narrow rangre of energies (typically $\pm 2 \%$ ) for subsequent analysis. The images recorded in both the sample ancd reference arms were analyzed (after subtracting a null image to correct for background) by dividing the images into a set of annuli and summing the intensity of the pixels over a sufficient number of annuli to obtain good signal to noise and self-consistency in the data. This typically required less than $1 \%$ of the : sample area to be taken for the central intensity, but a larger area could be taken if necessary, and the meeasured $n_{2}$ corrected by the expected dependence on sample area. ${ }^{3,5}$ In principle, the Z-scan curve is obtiained by taking the ratio of the central intensity in the sample and reference arms. We generally found, howwever, that the signal to noise ratio of the Z-scan curve was better if we took the ratio of the integrated cemaral intensity to the total integrated intensity in the image, which eliminated effects of gain fluctuations bestween the two cameras and frame grabbers. The ratio of the integrated intensities of the full beams in tine sample and reference arms was checked to make sure that there was no observable central dip caused by two-photon absorption. At shorter wavelength, where this becomes important, it would be possible to deconvolve both effects from the data.

\section{Numerical Calculations}

The calculated beam profiles presented in Fig. 4, as well as other resui-ss presented here, were performed with a commercial personal computer version (GLAD) of a diffractive propagation code originally developed for Los Alamos. ${ }^{7}$ This code decomposes an input beam into an array of Fourier components, propagates each component over a specified distance, and perferms an inverse Fourier transform to reconstitute the beam at its new location. Linear and nonlinear coptical elements may be placed in the beam at each location to alter its phase distribution. A split-step metnod may also be used to account for phase changes through an extended nonlinear medium. For a more : detailed discussion, we refer the reader to a recent publication 8 that presented similar calculations. As sseen from Fig. 3 , sharp changes in the beam profile are not fully resolved because of the limitation on arrray size and the need to provide a large guard band around the beam to prevent Fourier aliasing. The coalculation in Fig. 3 was performed with a $512 \times 512$ array; consistent results in amplitude changes were ocotained with array sizes of $256 \times 256$ or greater.

An interesting (and surprising) feature of Fig. 3(b), which was calculatec: assuming a negligibly thin sample, is that there is essentially no energy $(<0.3 \%$, possibly due to calculational accuracy limitations) transferred outside the original image, despite large changes in the ereergy distribution within the image. This result begins to break down for a thick sample, or for a beam thaat is not a perfect flat top at the sample, but the energy in the wings remains small.

The relay-imaged experiments performed at $1064 \mathrm{~nm}$ were carried out wwith a beam that was a truncated gaussian rather than a flat-top beam, obtained by expanding the bear utilized for gaussian Z- 
scan experiments by a factor of 5 to a waist $\mathrm{w}=11 \mathrm{~mm}$ and aperturing it at $\sim 81 \%$ of the peak with an aperture of diameter $d=7.1 \mathrm{~mm}$. This aperture size was chosen to give approximately the same beam waist and confocal parameter as in the gaussian experiments $(\mathrm{d} \approx \pi \times 2.2 \mathrm{~mm})$. Calculated results for such an input beam, presented in Fig. 4 and summarized in Table 1, show that there is only a $4 \%$ decrease in sensitivity for this apertured gaussian beam as compared to the flat top. This allows considerably more of the beam energy to be utilized than if the beam has to be expanded sufficiently to approximate a flat top.

\section{Table I}

\section{Comparison of calculated Z-scan amplitudes for different input profiles} Gaussian Top-Hat Truncated Gaussian Astig. Gaussian (Apertured at $81 \%$ )

$\begin{array}{lcllc}\mathbf{I}_{\text {input }}\left(\mathrm{r}=0, \mathrm{MW} / \mathrm{cm}^{2}\right) & 50.0 & 3.0 & 3.0 & 50.0 \\ \text { Waist, } \mathbf{w}_{\mathbf{1}}(\mathrm{cm}) & 0.22 & 0.71 \text { (dia.) } & 1.1 \text { with } 0.71 \text { dia. } & 0.22,0.20 \\ \text { Energy }(\mathrm{mJ}) & 1.90 & 0.578 & 0.471 & 1.73 \\ \mathbf{I}_{\text {focus }}\left(\mathrm{r}=0, \mathrm{GW} / \mathrm{cm}^{2}\right) & 55.2 & 21.2 & 18.9 & 45.3 \\ \Delta \mathbf{T}_{\mathbf{p v}} & 0.356 & 0.342 & 0.294 & 0.298 \\ \Delta \Phi=(2 \pi / \lambda)\left(\gamma \mathbf{I}_{\text {focus }} L\right) & 0.880 & 0.337 & 0.301 & 0.722 \\ \Delta \mathbf{T}_{\mathbf{p v}} / \Delta \Phi & 0.405 & 1.014 & 0.975 & 0.413\end{array}$

Calculated for: $\lambda=1064 \mathrm{~nm}, \mathrm{f}=43 \mathrm{~cm}, \gamma=2.7 \times 10^{-16} \mathrm{~cm}^{2} / \mathrm{W}, \mathrm{L}=1.0 \mathrm{~cm}$

As discussed in the experimental section below, the gaussian Z-scan results typically gave somewhat lower values for $n_{2}$ than the truncated gaussian results. Calculations were performed to try to account for the effect on the Z-scan of the deviation from a perfect gaussian as illustrated in Fig.1. Several sources of possible deviation were explored. For example, a spherical aberration of -0.1 wave at the $1 / \mathrm{e}^{2}$ radius (i. e., a quadratic phase term that attained this value at $w_{o}$ ) gave approximately the observed amount of deviation from a perfect gaussian but yielded an increased sensitivity that very nearly canceled the decreased intensity at the focus. The ratio of peak to valley in this case became quite asymmetric, and the calculated Z-scan curve did not resemble any of the experimental data. A second source of deviation explored was simply to aperture the gaussian at a diameter near $3 w_{1}$, as was in fact the case experimentally. This gave a significant change in the focal size, and a correspondingly lower focal intensity (after renormalizing the input intensity to maintain constant energy throught the aperture). However, the increased sensitivity again nearly canceled any effect on the measured value of $n_{2}$. The basic reason for this, we believe, is that the beam size is only slightly altered at the peak and valley positions of the Z-scan. The largest effect we have calculated was to include a slight astigmatism in the beam, consistent with Fig. 1, as well as an aperture of $3.2 \mathrm{~mm}$ radius. The astigmatism was incorporated by taking the input beam waist in the vertical direction to be $10 \%$ smaller than the horizontal direction and adding a cylindrical lens of $100-\mathrm{m}$ focal length to the latter, yielding a 3-mm difference in focal position and the behavior shown in Fig. 5(a) similar to that of Fig. 1. In this case, the use of Eq. 2 
underestimates the value of $n_{2}$ obtained from the calculated Z-scan curve (Fig $5(\mathrm{~b})$ ) by $\sim 9 \%$. These comparisons were made by using $\Delta \Phi$ derived from the calculated focused intensity and the sensitivity $\Delta T / \Delta \Phi$ obtained from the Z-scan calculation.

If the sample is not thin compared to $z_{o}$, one must also account for the effect of sample thickness in order to extract an accurate value of $n_{2}$. In the gaussian beam case, this correction has been shown 9,10 to be relatively small provided the sample length does not exceed the confocal parameter within the sample, $z_{s}=n_{o} z_{o}$. For our case of a $1.3-\mathrm{cm} z_{o}$ and samples that ranged up to $1.3 \mathrm{~cm}$ in length, this correction is less than $3.3 \%$. This correction factor is expected to be somewhat larger for the top-hat case because the $\mathrm{Z}$-scan curve is sharper and in fact has a sign reversal beyond the region where the on-axis intensity goes through zero: $z= \pm 2 \mathrm{f}^{2} \lambda(\mathrm{d} / 2)^{2} .11$ Numerical calculations, performed with the GLAD code by splitting the sample into 2-mm slices and alternately applying self-focusing and propagating the beam, yield up to a $6 \%$ correction for the top-hat data. The accuracy of this calculation is still being verified.

\section{Experimental measurements}

The experiments were performed using a pulse sliced from a Q-switched single-longitudinal-mode Nd:YAG laser using a fast Pockels-cell driven by a nominally 500 -ps-wide pulse from an avalanche transistor high-voltage pulser having $<100 \mathrm{ps}$ rise and fall time. This method was chosen for pulse generation because the pulse widths obtained in this way were very stable and measurable with existing electronics (60-ps risetime vacuum photodiode with 6- $\mathrm{GHz}$ oscilloscope, or streak camera). Many Zscan measurements have been made with much-shorter-duration mode-locked pulses, which require autocorrelation techniques to measure the pulse width and are not necessarily sensitive to asymmetries in pulse shape. Ideally one would prefer to have a perfectly square temporal profile, otherwise one must integrate over the different intensities present in the pulse, $I(t)=I f(t)$ to obtain the time-averaged effect of the nonlinearity. In the regime in which the magnitude of the nonlinear response remains proportional to the intensity, the measured (average) index change $\langle\Delta n(t)\rangle$ is related to the index change $\Delta n_{o}=\gamma I_{o}$ at the peak of the pulse by ${ }^{3}$

$$
<\Delta n(t)>=\frac{\int_{-\infty}^{\infty} \Delta n(t) I(t) d t}{\int_{-\infty}^{\infty} I(t) d t}=\gamma I_{0} \frac{\int_{-\infty}^{\infty} f^{2}(t) d t}{\int_{-\infty}^{\infty} f(t) d t} \equiv \gamma I_{0} F
$$

For a pulse that is gaussian in time, this yields $\langle\Delta n(t)\rangle=\Delta n_{o} / \sqrt{2}$. In the case of our Pockels-cellswitched pulses, the factor $F$ by which the sensitivity is reduced, as calculated by integrating a typical streak camera trace shown in Fig. 10 , is essentially the same, $F \approx 0.72$, resulting largely from a small tail in the Pockels cell drive pulse, plus a contribution from the finite rise and fall of the pulse. The photodiode traces displayed an even larger tail and consequently smaller $F \approx 0.65$. However, this is to be expected from the impulse response of these photodiodes, which show a low-level tail of. We have 
consequently used the 355-nm streak ca-esra result to analyze the $355-\mathrm{nm}$ data and to correct the 1064$\mathrm{nm}$ photodiode result, for which streak carcera data were not available. This introduces some uncertainty in the 1064-nm results, but we believe: $=$ is to be the appropriate procedure to use. The size of the (downward) correction to $n_{2}$, about $2(0) \%$, is larger than just the ratio of $F$ values, because an overestimate of the amount of energy in tree tail not only underestimates the sensitivity of the technique (because of the averaging in Eq. 3), but also underestimates the peak power of the pulse, which is extracted from the measured energy and poulse shape. Thus an overestimate of the amount of energy in the tail gives rise to two factors that lead to textracting too large a value of $n_{2}$ from the measurements.

This sensitivity to the knowledge of the pulse shape presents, we believe, the largest source of uncertainty in these measurements. Althoough the pulse shape of the Pockels cell driver is quite stable, small changes in the timing of the laser purise relative to the Pockels cell give slightly different ratios of energy in the tail to energy in the peak. SSuch timing shifts were not always monitored as closely as possible in our initial measurements at $106+\mathrm{nm}$, and may explain some of the spread in measured values. Other sources of uncertainty in the measurements are believed to be relatively small: energies were crosscalibrated on several calorimeters, including ones at Livermore, 12 and are believed to be valid within $\pm 2 \%$; spot sizes were measured from masenified images captured on a ccd camera calibrated against a reticle, and are believed accurate within $=: 1 \%$. Peak-to-valley ratios could be measured to within a few tenths of a percent out of typically 15 to $30: 3 \%$, or within $\pm 3 \%$ or better, provided the sample was of good optical quality. In the case of flat-top beams, we found that the theoretical Z-scan curve closely approximated the derivative of a gaussisan out to $z= \pm 1.7 \mathrm{zo}$ and thus the data could be fit to this functional form to obtain an accurate value :for the peak-to-valley transmission.. An example of such a fit to experimental data is shown in Fig. 7.

\section{Reesults and discussion}

The nonlinear index data we have obstained is presented in Table II. Data is presented at $1064 \mathrm{~nm}$ for a gaussian beam, analyzed with the astiggmatic results, and for a truncated gaussian, labeled "near tophat." The $355-\mathrm{nm}$ data was taken with a very nearly top-hat beam, but one which showed some astigmatism near the focus. This setup nets further improvement to eliminate this problem, and this data should be considered as only preliminary. The values of $n_{2}$ given for fused silica are averages from a number of samples, including Corning 79.40, Dynasil 1000, Suprasil II and fused quartz, for which no statistically significant differences were seeen, but which ranged up to $6 \%$ higher and lower for the top-hat measurements and up to $10 \%$ for the gausssian measurements. The $1064-\mathrm{nm}$ value of $0.67 \pm 0.15 \times 10^{-13}$ esu is, however, about $30 \%$ lower than the accepted value of $0.95 \pm 0.1^{13,1}$ or recent measurements that have been performed in fibers ${ }^{14}$ or by the irequency-resolved optical gating technique. ${ }^{15}$ Measurements obtained by the gaussian Z-scan technique were in substantial agreement with the top-hat measurements when analyzed using the apertured astigmaraic calculations. These results are also in reasonable agreement with other Z-scan measurements that have recently been performed, which yielded a value of $0.74 \pm 0.15$ $\times 10^{-13}$ esu for fused silica at $1064 \mathrm{~nm}$. 16 we do not at present understand why the Z-scan technique has yielded consistently lower results for fuse-i silica than other methods. Our value for BK-7 is also lower 
than previously measured ${ }^{13}$ by an even larger ratio. On the other hand, our 1064-nm values for KDP, while somewhat lower than the previous measurement of $1.0 \pm 0.3 \times 10^{-13} \mathrm{esu},{ }^{13}$ are well within the stated errors and our values for the laser glasses LHG-8, LG-750, LG-770 are in the range of previous measurements of phosphate laser glasses. ${ }^{17,2}$

Our $355-\mathrm{nm}$ value for fused silica of $0.92 \pm 0.21 \times 10^{-13}$ esu shows an expected increase toward short wavelength and is is slightly higher than the value of $0.85 \pm 0.17 \times 10^{-13}$ esu given in Ref. 16, but lower than preliminary results obtained by frequency resolved optical gating. ${ }^{15}$ We know of no other measurement with which to compare our 355-nm value for KDP. This measurement had a larger uncertainty owing to some experimental problems and should be repeated.

\section{Table II}

Measured values of $\gamma$ and corresponding $\mathbf{n}_{2}$

1064 nm:

$\mathrm{n}_{\mathrm{o}} \quad \gamma\left(10^{-16} \mathrm{~cm}^{2} / \mathrm{W}\right) \quad \mathrm{n}_{2}\left(10^{-13} \mathrm{esu}\right)$

$\begin{array}{lllll}\text { Fused silica } & 1.45 & 1.92 \pm 0.4 & 0.67 \pm 0.15 & \text { (near top-hat) } \\ & & 1.94 \pm 0.4 & 0.68 \pm 0.15 & \text { (gaussian) } \\ \text { LHG-8 } & 1.53 & 2.92 \pm 0.6 & 1.07 \pm 0.22 & \text { (near top-hat) } \\ \text { LG-750 } & 1.53 ? & 2.93 \pm 0.6 & 1.08 \pm 0.22 & \text { (near top-hat) } \\ \text { LG-770 } & 1.53 ? & 2.69 \pm 0.6 & 0.99 \pm 0.22 & \text { (near top-hat) } \\ & & 3.10 \pm 0.7 & 1.14 \pm 0.26 & \text { (gaussian) } \\ \mathrm{BK}-7 & 1.51 & 2.23 \pm 0.5 & 0.81 \pm 0.18 & \text { (near top-hat) } \\ \mathrm{KDP}(\mathrm{e}) & 1.46 & 2.31 \pm 0.5 & 0.81 \pm 0.18 & \text { (near top-hat) } \\ \mathrm{KDP}(\mathrm{o}) & 1.49 & 2.57 \pm 0.6 & 0.92 \pm 0.22 & \text { (near top-hat) } \\ & & 2.55 \pm 0.6 & 0.91 \pm 0.22 & \text { (gaussian) } \\ \mathrm{KD} * \mathrm{P}(\mathrm{o}) & 1.46 & 2.94 \pm 0.7 & 1.05 \pm 0.25 & \text { (near top-hat) } \\ & & 2.96 \pm 0.7 & 1.06 \pm 0.25 & \text { (gaussian) } \\ \mathrm{KD} * \mathrm{P}(\mathrm{oe}) & 1.47 & 2.63 \pm 0.6 & 0.93 \pm 0.21 & \text { (near top-hat) } \\ \mathrm{KBr} & 1.54 & 5.89 \pm 1.5 & 2.18 \pm 0.55 & \text { (gaussian) }\end{array}$

\section{5 nm}

$\begin{array}{lllll}\text { Fused silica } & 1.47 & 2.62 \pm 0.6 & 0.92 \pm 0.21 & \text { (top-hat) } \\ \text { KDP(o) } & 1.53 & 5.60 \pm 2 & 2.06 \pm 0.7 & \text { (top-hat) }\end{array}$

The values reported here are still subject to further refinements, including more accurate calculations of the effect of sample thickness on the top-hat Z-scan, and are subject to uncertainties mentioned in respect to the pulse shape measurements. We do not expect that these effects could change the reported values by more than $10 \%$, unless some source of systematic error is identified. Errors quoted are estimated from the range of measured values and the uncertainties in experimental parameters. We believe the technique is inherently capable of highly accurate measurements if all of the experimental parameters (particularly accurate knowledge of the pulse shape) are carefully controlled. 


\section{Acknowledgments}

This work was performed under the auspices of the US Department of Energy and was supported by the Lawrence Livermore National Laboratory. We are grateful to David Eimerl and David Milam for many helpful discussions and to Robert Harrison for technical assistance.

\section{References}

1. R. R. Adair, L. L. Chase, and S. A. Payne, "Dispersion of the nonlinear refractive index of optical crystals," Optical Materials 1, 185 (1992); "Nonlinear refractive index of optical cryastals," Phys. Rev. B 39, 3337 (1989).

2. A. N. Azerenko, G. B. Al'tshuler, N. R. Beleshenkov, and S. A. Kozlov, "Fast nonlinearity of the refractive index of solid-state dielectric active media," Quantum Electron. 23, 633 (1993).

3. M. Sheik-Bahae, A. A. Said, T. H. Wei, D. J. Hagan, and E. W. Van Stryland, "Sensitive measurements of optical nonlinearities using a single beam," IEEE J. Quantum Electron. 26, 760 (1990).

4. D. Weaire, B. S. Wherrett, D. A. B. Miller, and S. D. Smith, "Effect of low power nonlinear refraction on laser-beam propation in InSb," Opt. Lett. 4, 331 (1979).

5. W. Zhao, P. Palffy-Muhoray, "Z-scan technique for top-hat beams," Appl. Phys. Lett. 63, 1613 (1993).

6. M. Born and E. Wolf, Principles of Optics, Pergammon, Secs.8.5, 8.8

7. Applied Optics Research Co. For identification only; mention of this product does not imply endorsement of this product over any similar products that may be available from other suppliers.

8. S. Hughes, J. M. Burzler, G. Spruce, and B. S. Wherrett, "Fast Fourier transform techniques for efficient simulation of Z-scan measurements," JOSA B 12, 1888 (1995).

9. M. Sheik-Bahae, A. A. Said, D. J. Hagan, M. J. Soileau, and E. W. Van Stryland, "Nonlinear refraction and optical limiting in thick media," Opt. Eng. 30, 1228 (1991).

10. J, A. Hermann and R. G. McDuff, "Analysis of spatial scanning with optically thick nonlinear media," JOSA B 10, 2056, 1993; P. B. Chapple, J. Staromylinska, and R. G. McDuff, "Z-scan Studies in the thin- and thick-sample limit," JOSA B 11, 975 (1994).

11. Ref. 6, Sec. 8.8. This distance is incorrectly stated, following Eq. 26, in early editions of this text, but follows from the derivation.

12. We are grateful to David Milam for checking our calibration against Livermore calorimeters.

13. D. Milam and M. J. Weber, "Measurement of nonlinear-index coefficients using time-resolved interferometry: Application to optical materials for high-power neodymium lasers," J. Appl. Phys.47, 2497 (1976).

14. K. S. Kim, R. H. Stolen. W. A. Reed, and K. W. Quoi, "Measurement of the nonlinear index of silica-core and dispersion-shifted fibers," Opt. Lett. 19, 257 (1994); T. Kato, Y. Suetsugu, M Takagi, E. Sasaoka, and M. Nishimura, "Measurement of the nonlinear refractive index in optical fiber by the cross-phase-modulation method with depolarized pump light," Opt. Lett. 20, 988 (1995).

15. A. J. Taylor, T. C. Clement, and G. Rodriguez, personal communication.

16. R. DeSalvo, A. A. Said, D. J. Hagan, E. W. Van Stryland, and M.Sheik-Bahae, "Infrared to 
ultravicieet measurements of 2-photon absorption and $n_{2}$ in wide bandgap solids," to be published.

17. 2. M Lilam and M. J. Weber, "Nonlinear refractive index coefficient for Nd phosphate laser glasses. IEEE J. Quantum Electron. 12, 512 (1976). 


\section{Figure captions}

Fig. 1. Typical measured beam waist size as a function of distance from the focus. The curves show the best fit to gaussian beam propagation, $w=w_{0}\left(1+z^{2} / z_{0}^{2}\right)^{1 / 2}$. The horizontal and vertical focal positions differ by $3 \mathrm{~mm}$.

Fig. 2. Experimental setup for relay-imaged top-hat Z-scan. The defining aperture for the top hat is placed at the front focal plane of the focusing lens and imaged onto the ccd camera by a second lens.

Fig. 3. Calculated radial intensity distribution on the ccd camera, a) without, and b) with relay imaging. In (a), the aperture is placed directly before the focusing lens and the second lens is removed. In (b), the intensity distribution at the peak and valley of the Z-scan curve in the presence of a nonlinear medium is also shown. Similar increases and decreases of the intensity distribution are obtained for (a), but the distributions are modulated by the Fresnel ring structure shown.

Fig. 4. Comparison of calculated Z-scan curves for a gaussian beam, a top-hat beam, and a gaussian beam truncated at $81 \%$ of its peak for the beam parameters given in Table $\mathrm{I}$.

Fig. 5. a) Calculated waist sizes for a slightly astigmatic beam with the parameters given in Table $I$, in an attempt to model the experimental behavior in Fig.1. The beam is also apertured at a radius of $3.2 \mathrm{~mm}$. b) Z-scan curve calculated for these beam parameters. A slight broadening of the Z-scan curve compared to the gaussian of Fig. 4 is evident.

Fig. 6. Temporal profile of the $355-\mathrm{nm}$ pulse measured on a streak camera.

Fig. 7. Fit of Z-scan data for fused silica obtained with a near top-hat truncated gaussian at $1064 \mathrm{~nm}$ to the derivative of a gaussian. 


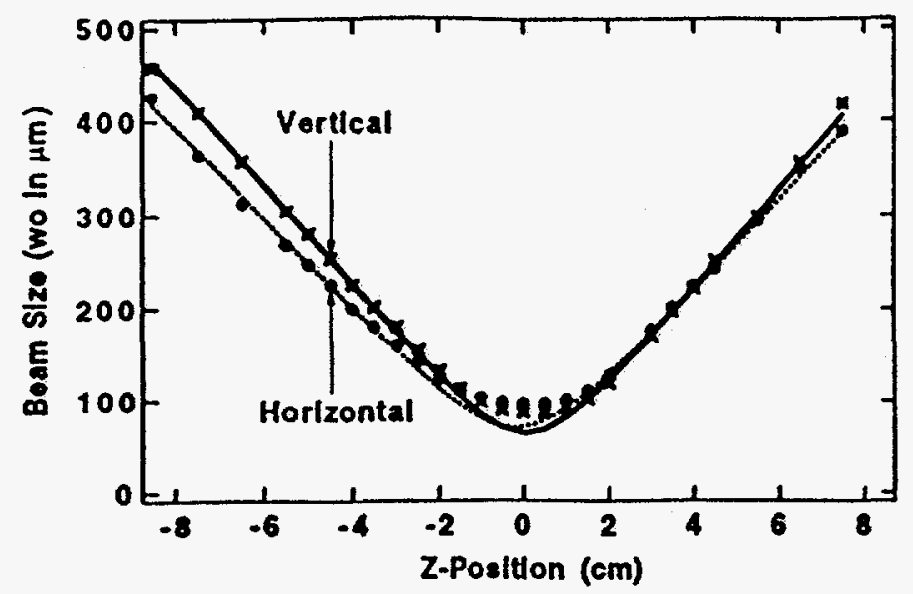

Figure 1

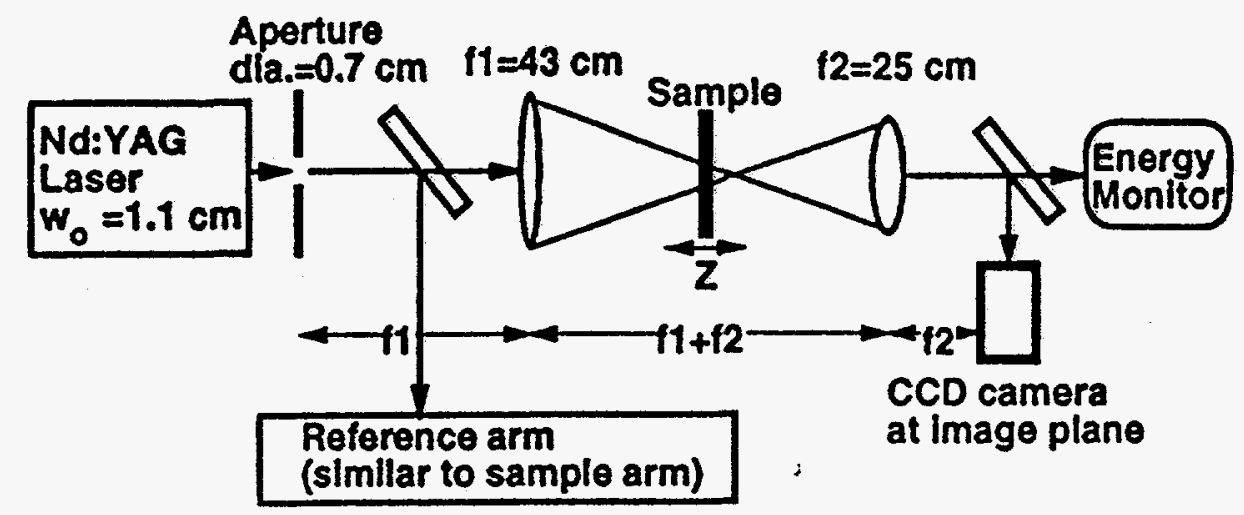

Figure 2
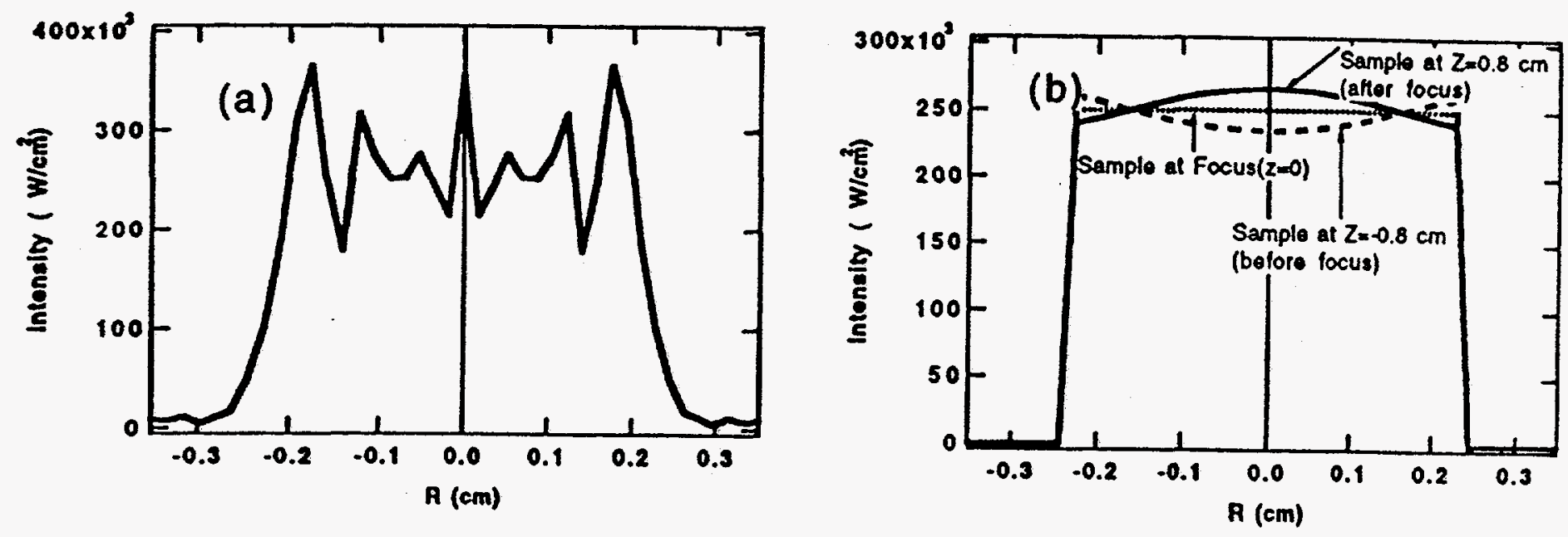

Figure 3 


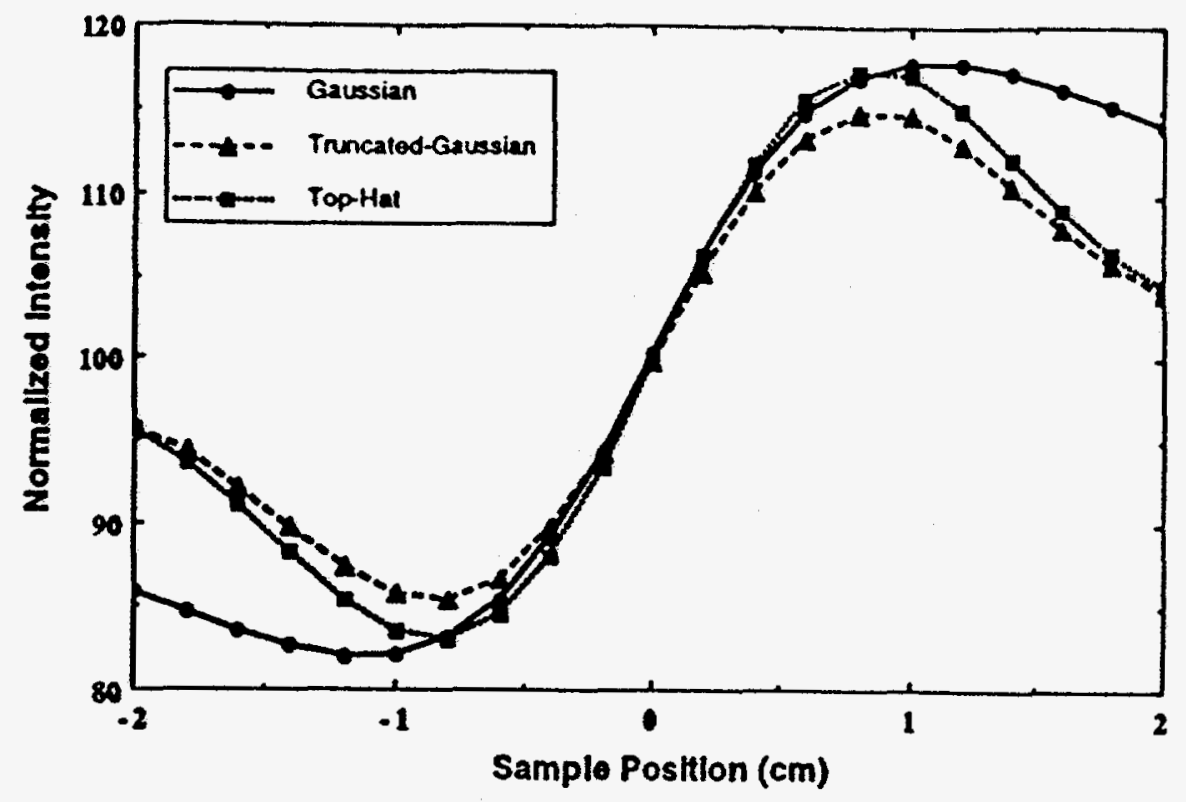

Figure 4
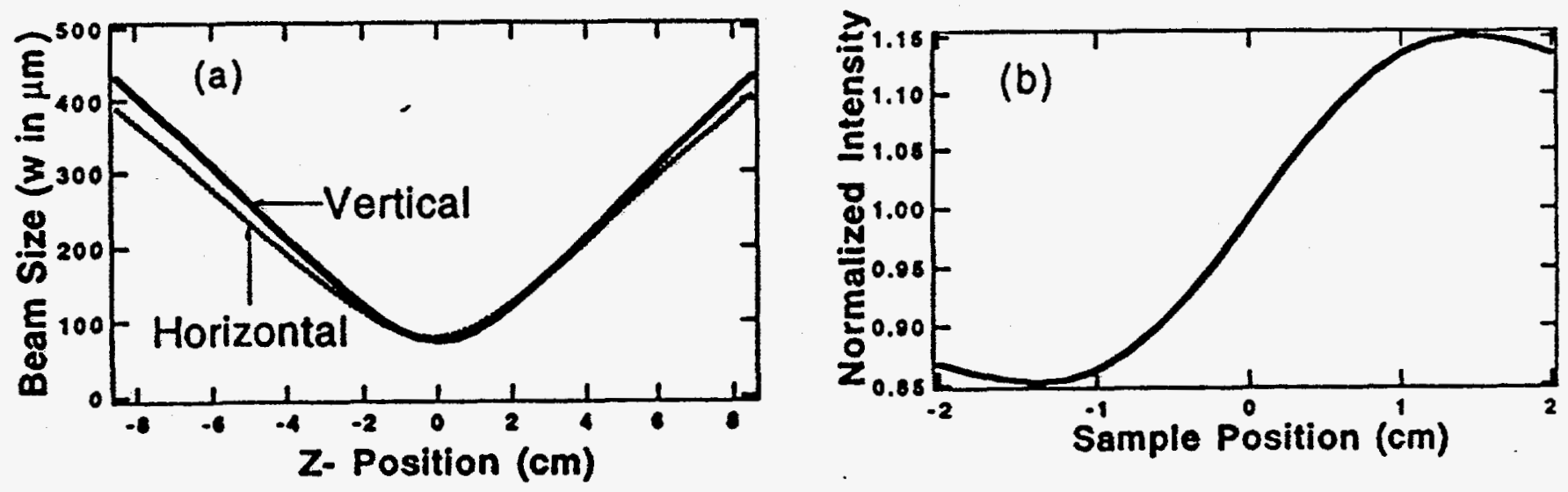

Figure 5 


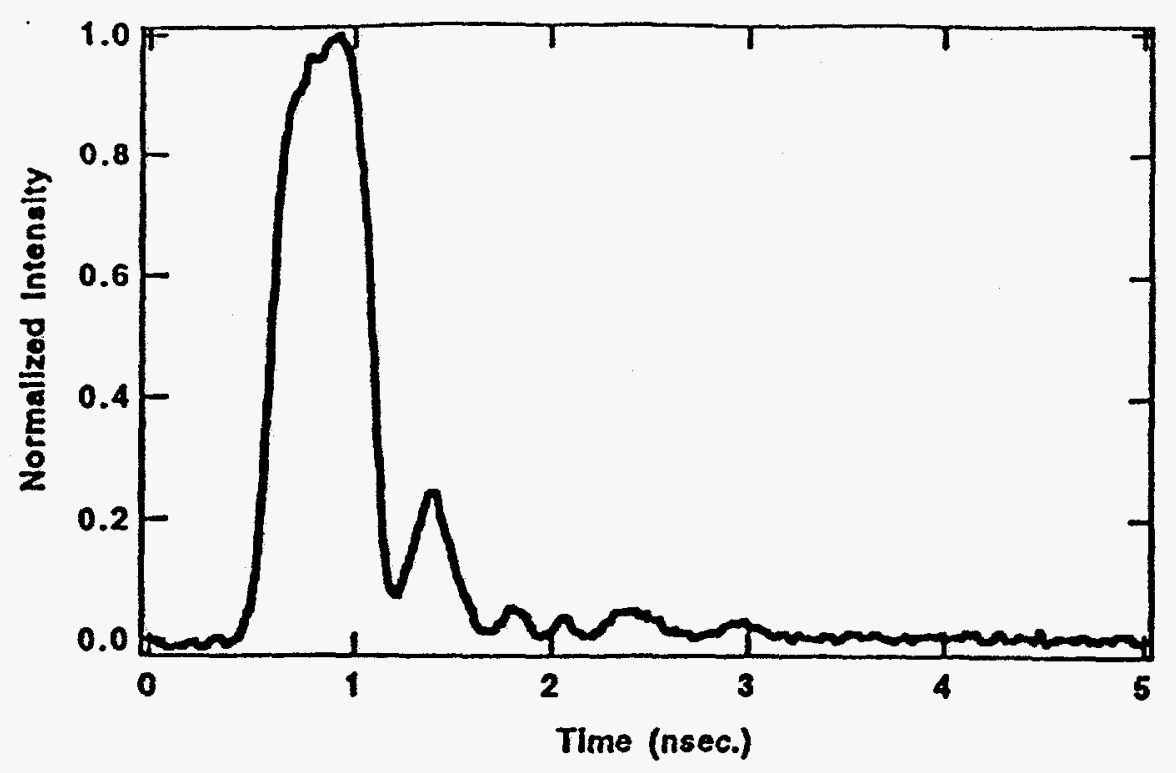

Figure 6

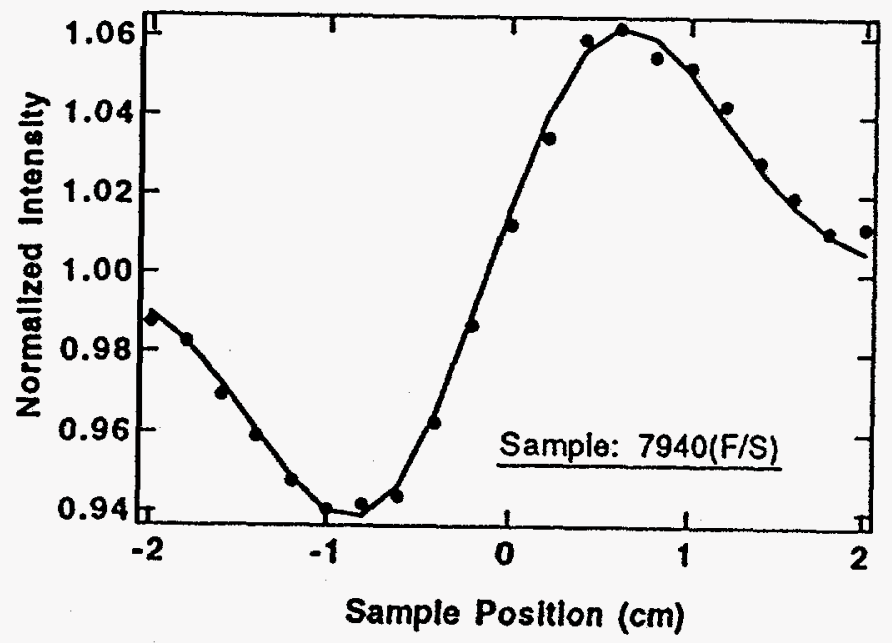

Figure 7

\section{DISCLAIMER}

This report was prepared as an account of work sponsored by an agency of the United States Government. Neither the United States Government nor any agency thereof, nor any of their employees, makes any warranty, express or implied, or assumes any legal liability or responsibility for the accuracy, completeness, or usefulness of any information, apparatus, product, or process disclosed, or represents that its use would not infringe privately owned rights. Reference herein to any specific commercial product, process, or service by trade name, trademark, manufacturer, or otherwise does not necessarily constitute or imply its endorsement, recommendation, or favoring by the United States Government or any agency thereof. The views and opinions of authors expressed herein do not necessarily state or reflect those of the United States Government or any agency thereof. 